\title{
Synthesis of nitrogen-doped carbon nanofibers and its application for the electrochemical detection of dopamine
}

\author{
Chunyan Wang ${ }^{1,2, a}$, Xiaoqiu Liu ${ }^{1}$ and Xiaoe Wang ${ }^{1}$ \\ ${ }^{1}$ School of Food Engineering, Jinlin Engineering Normal University, Changchun, Jilin 130052, \\ China \\ ${ }^{2}$ State Key Laboratory of Electroanalytical Chemistry, Changchun Institute of Applied Chemistry, \\ Chinese Academy of Sciences, Changchun, Jilin 130022, China \\ wangcy@ciac.ac.cn
}

\begin{abstract}
Keywords: nitrogen-doped carbon nanofibers, electrospinning, post-treatment, electrochemical detection, dopamine

Abstract Nitrogen-doped carbon nanofibers (NCNFs) was synthesized by simple electrospinning, followed by the thermal treatment using urea as nitrogen source. The morphology and microstructure of CNFs were characterized by scanning electron microscopy (SEM) and transmission electron microscopy (TEM). The results indicate that the prepared NCNFs exhibited small diameter and rough surface. The NCNFs film could be directly used for the electrode modification and showed good catalytic activity toward the oxidation of dopamine (DA) and could be used for the determination of DA by cyclic voltammetry and differential pulse voltammetry with a wide linear range and low detection limit. The proposed method was successfully applied to the analysis of real sample.
\end{abstract}

\section{Introduction}

As a new kind of carbon nanomaterials, carbon nanofibers (CNFs) have attracted enormous interest due to its unique physical, chemical, mechanical and electrical properties and shows wide application in the fields of electrocatalysis and chem/biosensors [1]. In the recent years, nitrogen-doped carbon nanofibers (NCNFs) have attracted extensive attentions because of its large specific surface area, excellent electrical conductivity, good stability and high mechanical strength. NCNFs have been widely used for the construction of electrochemical sensors [2,3]. Presently, the introduction of nitrogen into the CNFs include two main methods. One is by directly introducing the nitrogen into CNFs during the synthesis process $[4,5]$; the other is by the post-treatment of CNFs in the nitrogen-containing atmosphere [6]. These method generally produce powdery materials, so an insulating binder was required to stick the samples onto the support materials which may be degrade the electronic properties and weaken the electrochemical performance of NCNFs [7]. On the contrary, electrospun NCNFs could be produced in films form which can be cut to the disk-shaped and directly and stably adhered to the surface of electrode. The as-prepared electrode is expected to retain its high catalytic activity of NCNFs .

In this work, we develop a simple and controllable approach to prepare NCNFs by pyrolyzing the electrospun CNFs and urea in nitrogen atmosphere. The urea and nitrogen were used for nitrogen doping and surface etching. By the post-treatment of electrospun CNFs, NCNFs exhibited rough and cellular surface, small diameter, implying that NCNFs have more edge plane defects and large electroactive surface area. Furthermore, NCNFs film could be directly used for the electrode modification. The resulting NCNFs modifying electrode showed good catalytic activity toward the oxidation of dopamine (DA) and can be used for the selective determination of DA in the presence of ascorbic acid (AA).

\section{Experimental}

Chemical and Solutions. All the reagents were of analytical grade and used without further purification. Polyacrylonitrile (PAN, $\mathrm{Mw}=150,000)$ and nafion solution $(5 \mathrm{wt} \%)$ were purchased from 
Sigma-Aldrich Co. (USA). N, N-Dimethylformamide (DMF) were obtained from Beijing chemical Co. (China). Dopamine (DA), ascorbic acid (AA) and urea were obtained from Beijing Dingguo Biotechnology Co., Ltd. (China). The double distilled water was used for solution preparation. Phosphate buffer solutions (PBS, $0.1 \mathrm{M}$ ) of different $\mathrm{pH}$ were prepared from stock solutions of $0.1 \mathrm{M}$ $\mathrm{H}_{3} \mathrm{PO}_{4}, \mathrm{NaH}_{2} \mathrm{PO}_{4}, \mathrm{Na}_{2} \mathrm{HPO}_{4}$ and $\mathrm{NaOH}$.

Apparatus. The scanning electron microscopy (SEM) experiment was carried out using a PHILIPS XL-30 ESEM at an accelerating voltage of $20 \mathrm{kV}$. The transmission electron microscopy (TEM) images were acquired with a TECNAI G2 with the accelerating voltage of $200 \mathrm{kV}$. Electrochemical Measurements were performed with a CHI 660 electrochemical analyzer (USA). A three-electrode system was used, where a glass carbon electrode (GCE, $d=3 \mathrm{~mm}$ ) modified with NCNFs film was used as the working electrode, a platinum wire as the counter electrode and a $\mathrm{Ag} / \mathrm{AgCl}(3 \mathrm{M} \mathrm{KCl})$ electrode as the reference electrode. Electrochemical impedance spectroscopy (EIS) was performed in $5 \mathrm{mM} \mathrm{Fe}\left[(\mathrm{CN})_{6}\right]^{3-} /^{4-}(1: 1)$ with $0.5 \mathrm{M} \mathrm{KCl}$ as supporting electrolyte, using an alternating current voltage of $5 \mathrm{mV}$ within a frequency range of $0.01-10^{5} \mathrm{~Hz}$. A equivalent circuit was used to fit the obtained impedance spectra. The electrochemical conditions for differential pulse voltammetry (DPV) were optimized at scan rate of $10 \mathrm{mV} / \mathrm{s}, 30 \mathrm{mV}$ pulse amplitude and $50 \mathrm{~ms}$ pulse width.

Synthesis of NCNFs film [8]. Firstly, the CNFs were synthesized by a direct carbonization method using PAN nanofibers as the carbon precursor. Typically, PAN nanofibers were made via an electrospinning technique by using homogeneous $10 \%$ wt. PAN solution in DMF. During the electrospinning process, the voltage was kept as $18 \mathrm{kV}$, the distance between the spinneret and the collector was kept as $14 \mathrm{~cm}$ and the injection rate is $1 \mathrm{~mL} \mathrm{~h}^{-1}$. Stabilization and carbonization of PAN fibers were completed in a high temperature furnace by the following steps: 1) $300^{\circ} \mathrm{C}$ annealing in air for $2 \mathrm{~h}$ to oxidize PAN partially; 2) heating up to $900^{\circ} \mathrm{C}$ under the heating rate of $10^{\circ} \mathrm{C} \mathrm{min}^{-1}$ and stayed at this temperature for $1 \mathrm{~h}$ for the shape stabilization and combination of the fibers, and then cooled to room temperature in nitrogen. Secondly, CNFs and urea were placed in a high temperature furnace with the mass ratio of $1: 10$. The carbonization was performed by heating up to $300^{\circ} \mathrm{C}$ for $1 \mathrm{~h}$ in highly pure nitrogen, then heating up to $900^{\circ} \mathrm{C}$ for $1 \mathrm{~h}$ with a heating-up rate of $10^{\circ} \mathrm{C} \mathrm{min}^{-1}$. Finally, it was cooled down to room temperature in nitrogen atmosphere.

Preparation of modified electrode. Prior to modification, GCE was polished with alumina slurry on a chamois leather, and then washed and ultrasonically cleaned in ethanol and redistilled water, respectively. Finally, the clean GCE was dried with $\mathrm{N}_{2}$. The NCNFs film was well cut out and then adhibited onto the clean GCE surface with $2 \mu \mathrm{L}$ of $0.5 \mathrm{wt} \%$ nafion solution. This modify method was convenient and effective compared that NCNFs was dissolved and casted on the GCE.

\section{Results and discussion}

Characteristics of NCNFs. The surface morphology of NCNFs was characterized by SEM. As shown in Fig. 1A, the NCNFs exhibited a diameter about $250 \mathrm{~nm}$ which loosely interwaved together and form a network-like structure. This structure is greatly enlarging electroactive surface area and facilitating the diffusion of analytes when applied to electrochemial reaction. Fig. 1B shows the typical TEM image of the as-prepared NCNFs, further exhibiting a rough and cellular surface implying that NCNFs has more edge plane defects and larger electroactive surface area because of surface etching and nitrogen doping.

Electrochemical characterization of NCNFs. The electrochemical performance of NCNFs-GCE was investigated by using $5 \mathrm{mM} \mathrm{Fe}\left[(\mathrm{CN})_{6}\right]^{3-/ 4-}$ as redox probe molecules. As shown in Fig. $2 \mathrm{~A}$, the response current at NCNFs-GCE is three times higher than that at GCE electrode, suggesting that NCNFs-GCE possess large electroactive surface area and numerous edge plane defects, which are favorable for its excellent electrocatalytic performance.

EIS can provide information on the impedance changes of the electrode surface during the modification process. Fig. 2B displayed the EIS results of the bare GCE and NCNFs-GCE, which were fitted by the equivalent circuit (Fig. 2B, inset). The results showed a notable decrease in resistance (Rct) 
at NCNFs-GCE compared with that at bare GCE, confirming that NCNFs were highly conductive and can accelerate electron transfer.
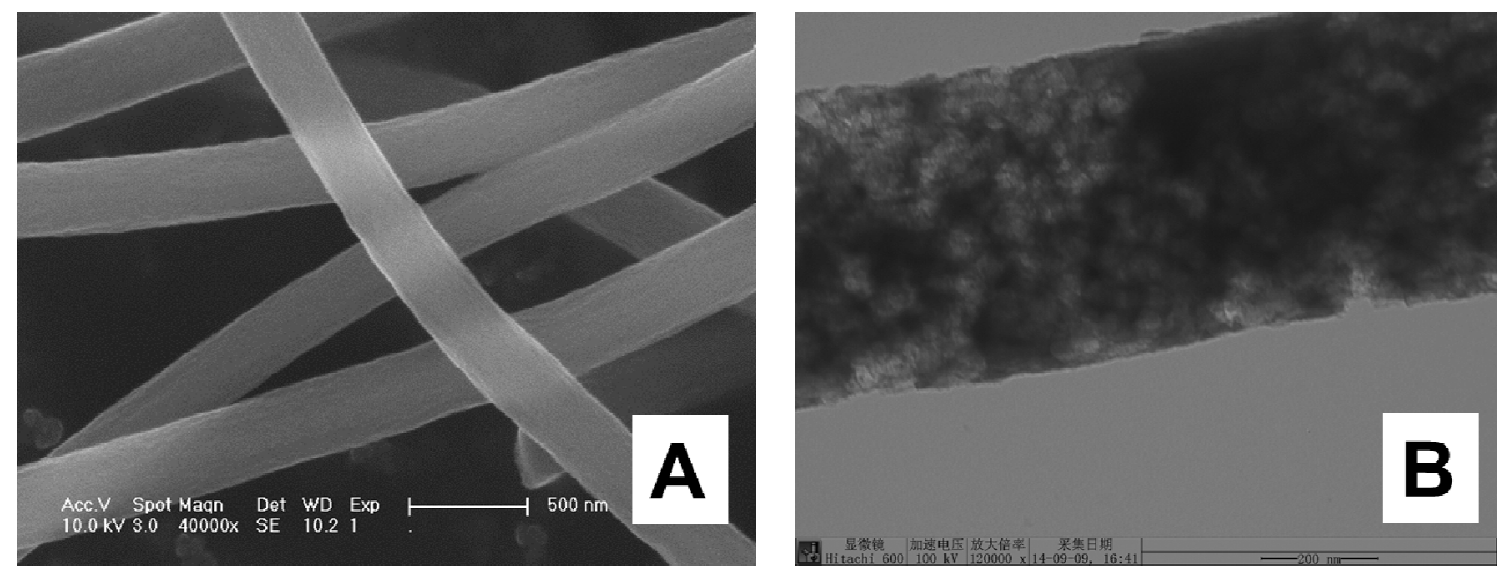

Fig. 1. (A) SEM and (B) TEM image of the NCNFs.
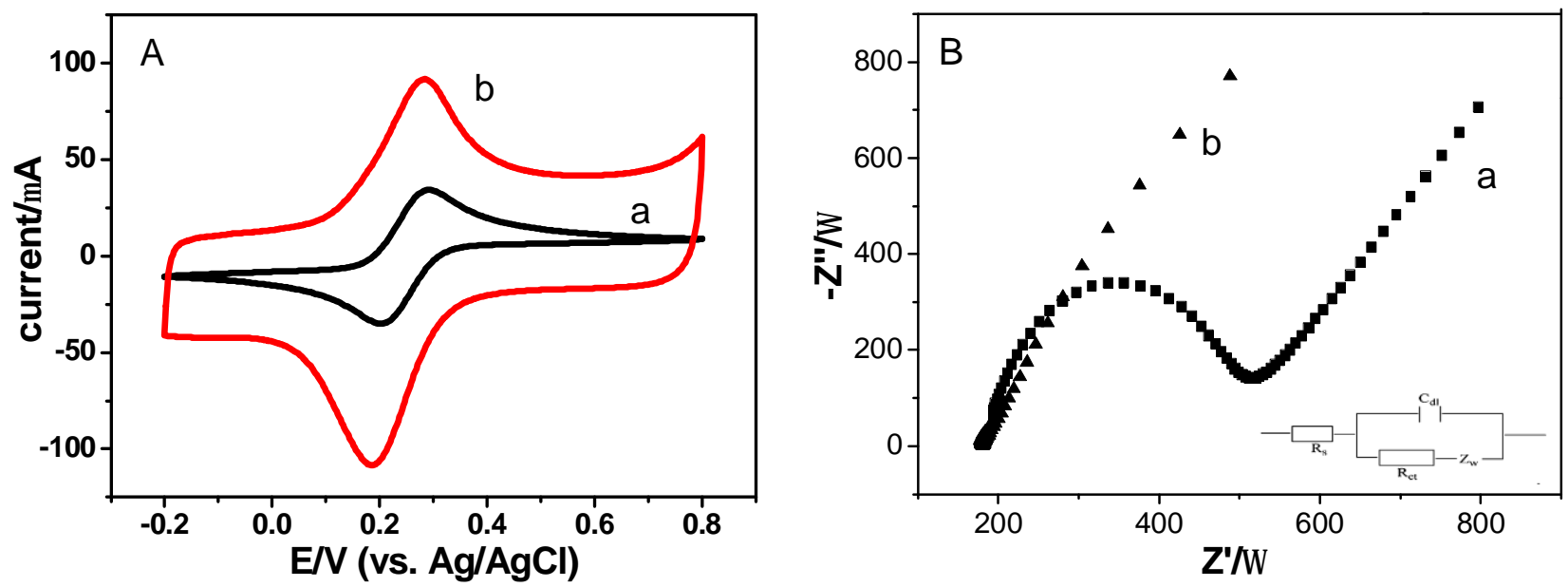

Fig. 2. (A) CVs of $5 \mathrm{mM} \mathrm{Fe}\left[(\mathrm{CN})_{6}\right]^{3-/ 4-}$ at GCE (a) and NCNFs-GCE (b). Electrolyte: $0.5 \mathrm{M} \mathrm{KCl}$; scan rate: $50 \mathrm{mV} \mathrm{s}^{-1}$. (B) Nyquist plots of EIS in the solution containing $5 \mathrm{mM}\left[\mathrm{Fe}(\mathrm{CN})_{6}\right]^{3-/ 4-}$ solution as probe and $0.1 \mathrm{M} \mathrm{KCl}$ as supporting electrolyte, inset is the equivalent circuit.

Electrochemical detection of DA. The electrochemical behavior of DA in $0.1 \mathrm{M} \mathrm{PBS}(\mathrm{pH} 7.0)$ at bare GCE and NCNFs-GCE electrodes was studied using cyclic voltammetry (CV). At the bare GCE, DA shows a pair of broad peaks with the anodic and cathodic peak potentials at 352 and $100 \mathrm{mV}$, respectively, indicating that the oxidation of DA at the bare GCE is irreversible electrochemical process and electron transfer kinetic process is sluggish (Fig. 3 a). While NCNFs-GCE provided it high electrochemical activities towards the oxidation of DA. The anodic peak potential negatively shifts to $251 \mathrm{mV}$, and the cathodic peak potential positively shifts to $170 \mathrm{mV}$ at NCNFs-GCE, indicating that the electrochemical reaction reversibility of DA was greatly improved (Fig. 3 b). The anodic peak current is 10-fold larger than that at bare GCE, suggesting that fast electron-transfer kinetics takes place at NCNFs-GCE. The improved electrochemical behavior of NCNFs-GCE may origin from the excellent properties of NCNFs. First, a large number of edge plane defects on NCNFs exhibited high electrocatalytic activities towards DA with lowered oxidation potential and increased peak current [9]. Second, due to good conductivity and unique electronic structure, NCNFs can accelerate electron-transfer as a promoter during the electrochemical reaction. Third, the network-like structure of NCNFs can improve the electroactive area and facilitate the transfer between the electrode and species in solution.

We further studied the determination of DA at NCNFs-GCE by DPV. Fig. 4 shows DPV responses of different concentrations of DA in $0.1 \mathrm{M}$ PBS ( $\mathrm{pH}$ 7.0). It seems that the anodic peak current is 
proportional to the concentration of DA. The linear range of DA is $1.5-220 \mu \mathrm{M}\left(\mathrm{r}^{2}=0.9990\right)$ with a detection limit (LOD) of $1.0 \mu \mathrm{M}(\mathrm{S} / \mathrm{N}=3)$. We also studied the selectivity of NCNFs-GCE by measuring the responses of DA in the presence of AA in $0.1 \mathrm{M}$ PBS ( $\mathrm{pH} 7.0)$ for six successive measurements. It is found that AA do not interfere with the determination of DA.

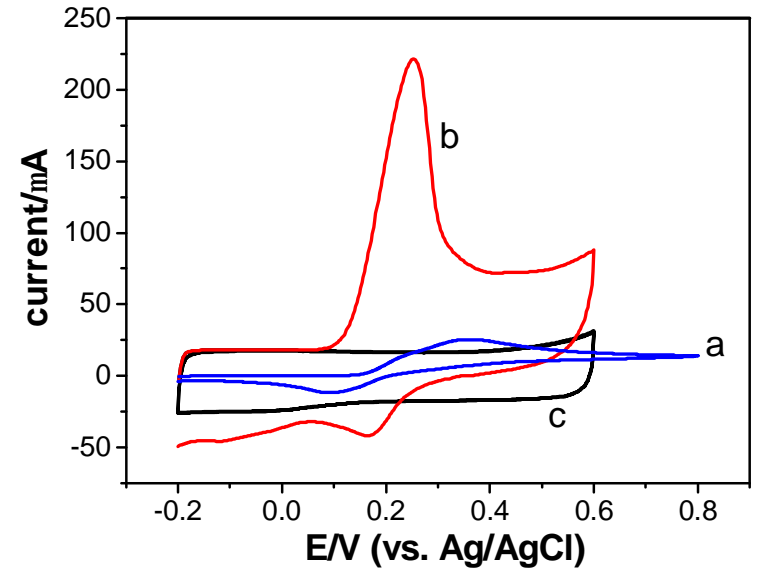

Fig.3. CVs for the bare GCE (a) and NCNFs-GCE (b) in $0.1 \mathrm{M}$ PBS containing $2.0 \mathrm{mM}$ DA, $\mathrm{c}$ is $\mathrm{CV}$ for the NCNFs-GCE $0.1 \mathrm{M}$ PBS. Scan rate: $50 \mathrm{mV} \mathrm{s}^{-1}$.

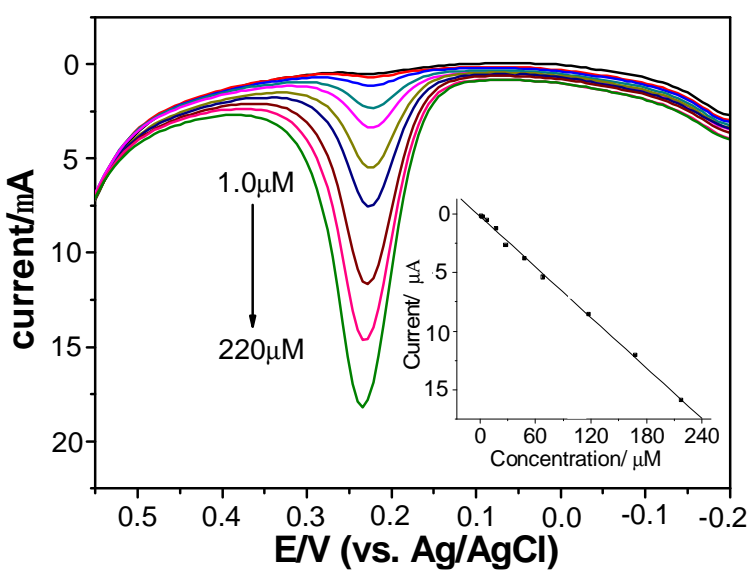

Fig. 4. DPV for various concentration of DA at the NCNFs-GCE in PBS solution at $\mathrm{pH}$ 7.0. The insert is the relationship between the peak current and the concentration of DA.

\section{Analytical applications.}

To investigate the practical applicability of the NCNFs-GCE for the determinations of DA, dopamine hydrochloride injection $\left(10 \mathrm{mg} \mathrm{mL}{ }^{-1}, 2 \mathrm{~mL}\right.$ per injection) was tested. It was diluted 10 times with double distilled water and no other pretreatment process was performed. The recovery experiments were performed by measuring the DPV responses to the samples in which the known concentrations of DA were added. The amounts of DA in the sample were then determined by calibration method and the recovery ranged from $97.4 \%$ to $106.8 \%$, which clearly indicated the NCNFs-GCE is feasible and reliable.

\section{Conclusions}

In this work, NCNFs were synthesized via a carbonization of the electrospun PAN nanofibers combined the pyrolyzation of the electrospun CNFs and urea. Owing to large specific surface area of NCNFs with large amounts of edge-plane-like defective sites, NCNFs-GCE was fabricated and used for the determination of DA. The NCNFs-GCE exhibits high sensitivity and enhanced reversibility for the oxidation of DA. The calibration curves was obtained in the range of $1.5-220 \mu \mathrm{M}$ and the detection limits was $1.0 \mu \mathrm{M}$. The NCNFs-GCE could be applied effectively to the detection of DA in injectable medicine with satisfying results.

\section{References}

[1] P. Serp, M. Corrias, P. Kalck. Appl. Catal. A 253 (2003), p.337-358.

[2] L. Jia, D.Bulushev, O. Podyacheva, et al. J. Catal. 307 (2013), p.94-102.

[3] D. Zhou, W. Li, X.Dong, et al. J. Mater. Chem. A 1 (2013), p.8488-8496.

[4] S. Maldonado, K. Stevenson. J. Phys. Chem. B 109 (2005), p.4707-4716.

[5] L. Chen, X. Zhang, H. Liang, et al. ACS Nano 6 (2012), p.7092-7102. 
[6] Y. Qiu, J. Yu, T. Shi, et al. J. Power Sources 196 (2011), p.9862-9867.

[7] H. Cong, X. Ren, P. Wang, et al. Energy Environ. Sci. 6 (2013), p.1185-1191.

[8] D. Liu, X. Zhang, T. You. Journal of Power Sources 273 (2015), p.810-815.

[9] K. Prasad, G. Muthuraman, J.M. Zen. Electrochem. Commun. 10 (2008), p.559. 